\title{
The Turning Point for Einstein's Annus Mirabilis*
}

\author{
Robert Rynasiewicz \\ Department of Philosophy \\ Johns Hopkins University \\ Baltimore, MD 21218 \\ Jürgen Renn \\ Max-Planck-Insitut für Wissenschaftsgeschichte \\ Wilhelm Str. 44 \\ 10117 Berlin
}

\begin{abstract}
The year 1905 has been called Einstein's annus mirabilis in virtue of three ground-breaking works completed over the span of a few months - the light quantum paper (Einstein, 1905a), the Brownian motion paper (Einstein, 1905c), and the paper on the electrodynamics of moving bodies introducing the special theory of relativity (Einstein, 1905d). There are prima facie reasons for thinking that the origins of these papers cannot be understood in isolation from one another. Due to space limitations, we concentrate primarily on the light quantum paper, since, in key respects, it marks the turning point for the annus mirabilis. The task is to probe, not just how the idea of the light quantum might have occurred to Einstein, but, more importantly, what convinced him that the idea was not just a quixotic hypothesis, but an unavoidable and demonstrable feature of radiation. The crucial development, we suggest, arose from comparing the energy fluctuations that following rigorously from the Stefan-Boltmann law, as well as from Wien's distribution formula for blackbody radiation, with what it is reasonable to expect from Maxwell's electromagnetic theory of light. A special case of this is addressed in (Einstein, 1904). The outcome for the general case leads naturally to the central theoretical argument of the light
\end{abstract}

\footnotetext{
*In loving memory of Jean Stern.

${ }^{\dagger}$ To appear in Studies in History and Philosophy of Modern Physics, March 2006.

${ }^{\ddagger}$ We would like to thank Olivier Darrigol, Clayton Gearhart, Michel Janssen, and John Norton for conversation and correspondence in connection with this paper.
} 
quantum paper, the expectation of Brownian-like motion, and several of the key results for the electrodynamics of moving bodies.

Keywords: Einstein; Light quantum; Blackbody radiation; Energy fluctuations; Brownian motion; Radiation pressure

\section{Introduction}

The annus mirabilis deserves its name for three ground-breaking papers on quantum theory, Brownian motion, and special relativity, respectively, that Einstein submitted for publication to the Annalen der Physik over the short span of three and a half months in the spring of 1905. Between the first and second of these papers he also produced a doctoral dissertation on a new method for determining atomic dimensions using fluid phenomena.

Much has been written about the genesis of these works, but by and large as though each had been produced independently of the others as outcomes of separate lines of research. As eclectic as Einstein's interests may have been, there are prima facie reasons for thinking that the origins of these papers cannot be understood in isolation from the others. For example, in his Autobiographical Notes, Einstein (1979) tells us why he chose to pursue physics rather than mathematics as a career. Whereas mathematics appeared to him to consist of a diversity of domains of detailed specialization, any one of which could consume an entire lifetime, in physics he learned early on to sort out what was incidental from what was truly essential - presumably with the concept of physics as a field with unified foundations. The Autobiographic Notes develops this theme of probing the foundations of physics during the decade 1895 through 1905, beginning with critiques of mechanics and electrodynamics, respectively, as inadequate, followed by attempts to construct a foundational alternative from known experimental facts, an endeavor that led to frustration and despair. Out of this emerged the annus mirabilis. The three papers completed in the spring of 1905 represent collectively a consolidation of lessons learned and insights gained in this failed enterprise. Each points to certain limitations of currently accepted physical laws. Each seeks to establish an inductively secured fixed point from which to carry on. Finally, each overlaps with at least one of the others in the details of the physics: for example, fluctuations play an essential role in both the light-quantum paper and the Brownian motion paper, while the empirical laws governing radiation are the concern of both the relativity paper and the light-quantum paper.

An account, even in outline, giving equal coverage to the origins of all three papers would require a monograph-length treatment. Here we focus primarily on the lightquantum paper, the one which Einstein singled out at the time as "very revolutionary." 1 Given his awareness of the iconoclasm of the view put forth, it took considerable nerve, and commensurate confidence, to put this paper together for publication. The decision to do so

\footnotetext{
${ }^{1}$ Einstein to Conrad Habicht, xx May 1905, CPAE, Vol. 5, Doc. xx.
} 
certainly marks a turning point, if not the turning point, not just for the annus mirabilis, but for Einstein's entire career. Our task then is to seek, not just how the idea of light quanta might have occurred to Einstein, but, more importantly, to explore what convinced him that this idea was not just a quixotic hypothesis, but an unavoidable and demonstrable feature of radiation that any candidate for a fundamental theory must account for. A major axis of these considerations involves a comparison of the energy fluctuations that follow rigorously from the Stefan-Boltzmann law for blackbody radiation with what it is reasonable to expect from Maxwell's electromagnetic theory of light. A special case of this is addressed in Einstein (1904). As Martin Klein recently remarked, here "one senses the lion showing his claws." ${ }^{2}$ With only a bit more effort, we suggest, one can also sense the lion grasping his prey. The outcome of the general case leads naturally to the central theoretical argument of the light-quantum paper and the expectation of Brownian-like motion, as well as to crucial groundwork for the electrodynamics of moving bodies.

\section{By the numbers}

Before proceeding, we would like to make a few remarks about method. For the project at hand, the external evidence, apart from its meagerness, offers up more in the way of puzzles than answers. This is so even for the one stretch of time - from late 1900 through early 1902 - for which there is a relative abundance of relevant correspondence due to the discovery of the "love letters" between Einstein and Mileva Marić. ${ }^{3}$ Thus, internal evidence - scrutiny of the structure and details of the papers in relation to one another and in relation to literature Einstein had studied - must bear an unusually heavy burden. Sometimes weight-bearing bonds form unexpectedly from propitious juxtaposition of materials. In other cases we have gone to considerable lengths to calculate the possibilities in the sense of exploring what results Einstein could have gotten from what with pencil and paper. To an extent, it is possible to build chains, however long, between arbitrarily chosen points. We have endeavored to constrain ourselves to chains with a single link. Also in this connection we have attempted, and urge others, to heed what we know about Einstein's characteristic style of thought. John Stachel's revised and expanded introduction to the second edition of The Miraculous Year (Einstein, 2005) provides an illuminating orientation. From it we borrow a few quotes and make a few additional comments.

It is indubitable to me, that our thinking occurs for the greatest part without the utilization of signs (words), and in addition also largely without consciousness ... It is in itself not necessary that a concept be attached to a sign (word) that is perceptible to and reproducible by the senses; however, if it is, then thinking thereby becomes communicable. Words or language, as they are written or spoken, do not seem to play any role in my mechanism of thought. The

\footnotetext{
${ }^{2}$ London, March 5, 2005.

${ }^{3}$ See (CPAE, Vol. 1) and (Einstein \& Marić, 1992).
} 
psychical entities which seem to serve as elements in thought are certain signs and more or less clear images which can be voluntarily reproduced and combined ... [T] he above mentioned elements in my case are of visual and some of muscular type. Conventional words or other signs have to be sought for laboriously only at a secondary stage ... (Einstein, 1954, pp. 25-26. Quoted in Stachel, 2005, p. xxxvii).

[Einstein] works more imaginatively and does not seem to trust the work the work we are doing in Göttingen; he has never thought on such formalistic lines. His powers of imagination are closely related to reality. He told me that he visualizes the gravitational waves with the help of an elastic body, and at the same time he made a movement with his fingers as though he were pressing an india rubber ball ... (Seelig, 1956, p. 155. Quoted in Stachel, 2005, p. xl).

Stachel summarizes:

For Einstein, the process of thinking was a solitary activity, primarily nonverbal in nature. At a secondary stage, it was necessary for him to transform the results of this primary process into forms communicable to others (Stachel, 2005. p. xxxv).

In conjunction with this, Stachel also develops Einstein's use of others as "sounding boards," particularly in his early years, in order to put his thoughts into communicable form.

We believe, however, that Stachel would agree that, for Einstein, the interaction between non-verbal and verbal processes of thought has a more ramified structure. As any good physicist knows, it is the numbers that come out that make or break the ideas or models from which they are derived, and good physics teachers preach to their students the importance of seeing their calculations through to the very end. If the rule has exceptions, Einstein is not one of them, at least as a practitioner (if not also as didact). Witness the role of the value of the precession of the perihelion of Mercury in his quest for a general theory of relativity as documented by Janssen $(1999,2003)$. There is every reason to think that various fundamental constants played an analogous role in the earlier period. It follows that even at the primary stage prior to communication there is a level at which thinking must be symbolic, namely, the level of explicit calculation. The assumptions and principles on which various equations are based may very well reside in the unarticulated realm of non-symbolic conceptualization. If a calculation yields unsuitable results, revision and adjustments at the pre-symbolic stage are necessary. Separate, successful calculations may require integration at the pre-symbolic stage of the ideas on which they were based. When integration is problematic, "sounding boards" may be useful for determining whether apparent conflicts are real or based on tacit, inessential presuppositions. Finally, when all is well, there is still the matter of recasting what has been achieved as a structured argument, 
or a sequence of structured arguments, that is compelling for the unconvinced. Apart from the clear articulation of the concepts and principles, this may require revised, reformulated, or even inverted derivations to suit the form of the argument.

Finally, into all this must be factored inadvertent computational errors that can lead to dead ends and force backtracking. These are familiar to those who have worked through Einstein's notebooks on the development of general relativity. ${ }^{4}$ It would be unusual were the years prior to 1905 free of such setbacks. We have direct evidence of at least one case. In a letter to Mileva Marić from December 1901, Einstein reveals:

I wrote to you that I doubted the correctness of the ideas about relative motion. My reservations rested, however, solely on a simple calculational error. Now I believe in them all the more ${ }^{5}$ (Einstein to Mileva Marić, 17 December 190, CPAE, Vol. 1, Doc. 128).

Obviously, without the surviving scratch pads, such detours can never be known.

\section{Overview of the 1905 light-quantum paper}

In this section, we register two observations about the content and structure of the lightquantum paper (Einstein, 1905a) in order to set the stage for what follows.

First is the central thesis of the paper. In the introduction Einstein formulates it as follows.

According to the assumption to be considered here, in the propagation of light from a point source, the energy is not distributed continuously over a space which grows larger and larger, but consists of a finite number of energy quanta localized at spatial points, which move without dividing and which can be absorbed and produced only as wholes(Einstein, 1905a, p. 133).

The title of the paper, however, reads "On a heuristic point of view concerning the production and transformation of light." In what sense are we to understand the point of view put forward as "heuristic"?

In $\S 2$ Einstein takes Planck's law,

$$
\varrho(\nu)=\frac{\alpha \nu^{3}}{e^{\beta \nu / T}-1},
$$

to be an empirically accurate description of the frequency distribution of the energy density $\varrho$ of blackbody radiation at absolute temperature $T$. In the remainder, Einstein concerns

\footnotetext{
${ }^{4}$ See (Renn, forthcoming).

${ }^{5}$ All subsequent translations are our own unless noted otherwise.
} 
himself only with "Wien radiation," that is, frequencies of thermal radiation for which Wien's superseded spectral law,

$$
\varrho(\nu)=\alpha \nu^{3} e^{-\beta \nu / T},
$$

is sufficiently accurate. The ratio of (2) to (1) converges quickly to unity as $\beta \nu / T$ increases, exceeding .95 already for $\beta \nu / T=3$. A comparison of the spectral curves, which are scale invariant in $\nu / T$, is given in figure 1 for $T=300 \mathrm{~K}$. The absolute difference

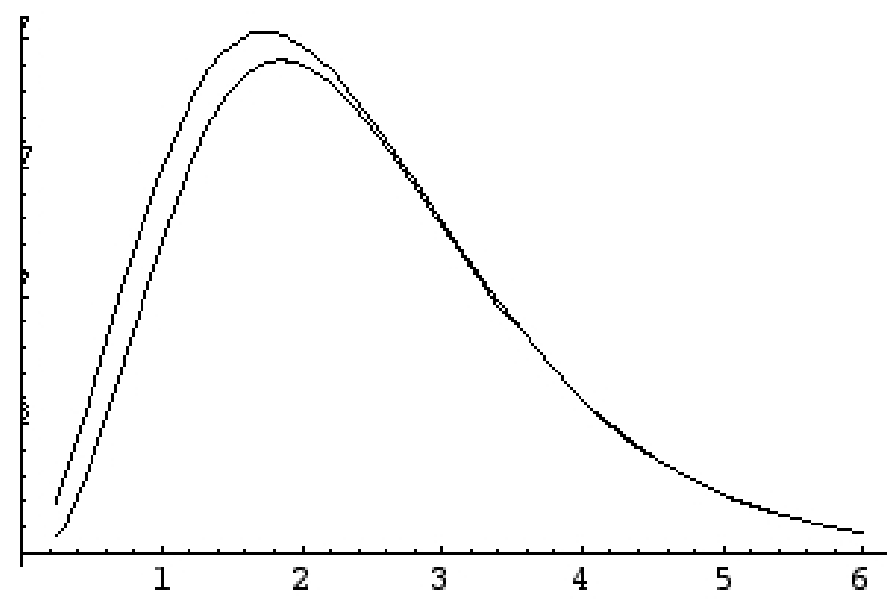

Figure 1: Planck spectrum (upper curve) vs. Wien spectrum (lower) for $T=3000 \mathrm{~K}$ with frequencies in units of $10^{14} \mathrm{sec}^{-1}$.

between the curves converges to zero with decreasing temperature. At the time they were experimentally distinguishable only for temperatures above $10^{3} \mathrm{~K}$. Thus, Wien's law is phenomenologically adequate over the entire spectrum for sufficiently low density (i.e., low temperature) radiation or for sufficiently high frequencies for arbitrarily high temperatures. Our understanding of the sense in which Einstein proposes the light-quantum picture as a heuristic point of view is that it is intended not as a thesis about the fundamental ontology of radiation, but rather, in the first instance, as a useful model for understanding the processes of the absorption, production, and propagation of light for certain regimes of electromagnetic radiation, and in the second, as a condition of adequacy for any future fundamental theory, i.e., that whatever the ultimate structure of radiation, it must behave as if it consists of independent light quanta in the Wien regime.

Our second preliminary observation addresses how the various sections of the lightquantum paper hang together. The paper has a highly modular structure, dividing into three parts entirely independent of one another. In outline form, the nine sections of the paper parse into three major divisions as follows. 
I. Negative theoretical arguments.

$\S 1$. The reductio of classical foundations.

$\S 2$. The irrelevance of Planck's theory for the

determination of fundamental constants.

II. The positive theoretical (entropic) argument.

$\S \S 3-6$.

III. Experimental consequences.

$\S 7$. Photoluminescence.

$\S 8$. Photo-electric effect.

$\S 9$. Ionization of gases.

Among the two negative theoretical arguments, the second builds in an essential way on the first. The sections concerning experimental consequences follow a hypothetico-deductive strategy: If light consists of independent light quanta, what predictions are expected for these respective phenomena? The central theoretical argument has a more inductive flavor: Given the relation between entropy and volume that can be derived from Wien's spectral law, what does this entail about the behavior of its spatial structure assuming that entropy is a monotonic function of the relative probability of the state of the system?

\section{The role of fluctuations in the entropic argument}

Einstein launches the chain of reasoning from thermodynamical properties of Wien radiation to its light-quantum structure by establishing by means of a straightforward variational argument in $\S 3$ that, whatever the spectral distribution of blackbody radiation the entropy density per unit volume expressed as a function $\varphi(\varrho, \nu)$ of energy density and frequency satisfies the relation

$$
\frac{\partial \varphi}{\partial \varrho}=\frac{1}{T}
$$

Thus, $\varphi(\varrho, \nu)$ can be explicitly computed by integration if $\varrho(\nu, T)$ is given. ${ }^{6}$ In $\S 4$ Einstein does so using Wien's spectral law, with the result:

$$
\varphi(\nu, T)=-\frac{\varrho}{\beta \nu}\left\{\log \frac{\varrho}{\alpha \nu^{3}}-1\right\} .
$$

He then reasons as follows. If $V$ is the total volume and $E$ the total energy corresponding to radiation lying in the frequency range from $\nu$ to $\nu+d \nu$, then, since $E=V \varrho d \nu$, the entropy of the radiation in this frequency slice is

$$
S=V \varphi(\varrho, \nu) d \nu=-\frac{E}{\beta \nu}\left\{\log \frac{E}{V \alpha \nu^{3} d \nu}-1\right\} .
$$

\footnotetext{
${ }^{6}$ Assuming that $\varphi=0$ when $\varrho=0$.
} 
Hence, argues Einstein, if $S_{0}$ is the entropy corresponding to a different volume $V_{0}$, then

$$
S-S_{0}=\frac{E}{\beta \nu} \log \left(\frac{V}{V_{0}}\right) .
$$

This, Einstein claims, shows that the entropy of monochromatic radiation of sufficiently small energy density varies with volume according to the same law as for an ideal gas or a dilute solution.

At this point, the attentive reader should be baffled. The law for an ideal gas (resp., dilute solution),

$$
S-S_{0}=m R \log \left(\frac{V}{V_{0}}\right)
$$

where $m$ is the number of moles of gas, is standardly understood to describe the change in entropy of the gas under a quasi-static isothermal compression (or expansion) from $V_{0}$ to $V$ involving a change in internal energy due to an exchange of heat. However, if the relation (6) for monochromatic blackbody radiation, is supposed to describe an isothermal compression (expansion) of a radiation space, then the radiation density $\varrho$ remains constant. The energy at volume $V_{0}$ is $E_{0}=\left(V_{0} / V\right) E$. Then, setting

$$
S_{0}=V_{0} \varphi(\varrho, \nu) d \nu=-\frac{E_{0}}{\beta \nu}\left\{\log \frac{E_{0}}{V_{0} \alpha \nu^{3} d \nu}-1\right\}
$$

one finds instead that

$$
\begin{aligned}
S-S_{0} & =\frac{E}{\beta \nu}\left(1-\frac{V_{0}}{V}\right)\left(1-\log \frac{E}{V \nu^{3} d \nu}\right) \\
& =\left(V-V_{0}\right)\left\{\log \frac{\varrho}{\alpha \nu^{3}}-1\right\},
\end{aligned}
$$

as one would expect. That Einstein treats the energy $E$ as though it were a constant in the transition from (5) to (6) suggests that he may have had in mind a slow adiabatic transition. In that case, though, we cannot speak of the same monochromatic radiation. Although the total energy over all frequencies remains fixed, the temperature undergoes a transition from $T_{0}$ to $T$, and hence the radiation energy density from $\varrho\left(\nu, T_{0}\right)$ to $\varrho(\nu, T)$. However, the equation $V \varrho(\nu, T) d \nu=V_{0} \varrho\left(\nu, T_{0}\right) d \nu$ does hold in general.

What, then? In $\S 5$ Einstein turns to the statistical foundations of thermodynamics, showing that if entropy of the state of a system is a monotonic function of its relative probability $W$, then it must be a linear function of the natural logarithm of $W$ in which the constant of proportionality, as determined by the kinetic theory of gases is $R / N$, where $R$ is the universal gas constant and $N$ is Avogadro's number. Thus, if $S_{0}$ is the entropy of the initial state and $W$ the relative probability of a state with entropy $S$, then

$$
S-S_{0}=\frac{R}{N} \log W,
$$


a relation which Einstein subsequently refers to as Boltzmann's principle. Consider now the kinetic model of an ideal gas: $n$ independent particles confined to a space $V_{0}$ with no preference for one direction or one part of the space over any other. Selecting one of these particles, the probability that it occupies a sub-volume $V$ of $V_{0}$ is just $V / V_{0}$. Since the particles are assumed to be independent of one another, the probability $W$ of finding all $n$ particles in the sub-volume $V_{0}$ is $\left(V / V_{0}\right)^{n}$. Thus,

$$
S-S_{0}=R\left(\frac{n}{N}\right) \log \left(\frac{V}{V_{0}}\right) .
$$

If the initial state is an equilibrium state, then $W$ in this equation is clearly the probability that all $n$ particles spontaneously come to occupy the sub-volume $V$. Thus, Einstein has shown that equation (7) also holds for non-equilibrium states arising from random fluctuations. This is the manner in which equation (6) for radiation entropy is to be understood. Specifically, (6) can be rewritten in conformity with Boltzmann's principle (9) as

$$
S-S_{0}=\frac{R}{N} \log \left(\frac{V}{V_{0}}\right)^{\frac{N}{R} \frac{E}{\beta \nu}} .
$$

And, according to the interpretation of Boltzmann's principle,

$$
W=\left(\frac{V}{V_{0}}\right)^{\frac{N}{R} \frac{E}{\beta \nu}}
$$

is the probability that, at any given moment, the entire energy of monochromatic Wien radiation of frequncy $\nu$, initially enclosed by reflecting walls in a volume $V_{0}$, will come to occupy only the sub-volume $V$. Thus, Einstein concludes, the radiation behaves as if it is composed of independent energy quanta of size $\frac{R}{N} \beta \nu$.

From here Einstein proceeds to explore the consequences of this heuristic for several phenomena involving the emission and absorption of radiation. For us, though, it is worthwhile to ponder a bit further the system under consideration. In order to standardize notation, let $E_{0}$ be total energy of the volume $V_{0}$ surrounded by reflection walls. Mentally carve the volume $V_{0}$ into two fixed sub-volumes $V$ and $V^{\prime}$. These are then adjacent systems in thermal contact. If $E$ is the momentary energy contained in $V$, then the average energy of $V$ is $\langle E\rangle=\left(V / V_{0}\right) E_{0}$. And, on average, the number of light quanta occupying $V$ is

$$
\langle n\rangle=\frac{E}{\frac{R}{N} \beta \nu} .
$$

The momentary value of the energy $E$ will fluctuate about $\langle E\rangle$ as more or fewer than $\langle n\rangle$ light quanta occupy $V$. Let

$$
\varepsilon={ }_{d f} E-\langle E\rangle \text {. }
$$

Then

$$
\left\langle\varepsilon^{2}\right\rangle=\left\langle E^{2}\right\rangle-\langle E\rangle^{2}
$$

This provides a first estimate of the scale of the energy fluctuations for $V$. 


\section{$5 \quad$ Fluctuations in the very small}

The penultimate section (§4) of (Einstein, 1904) derives the formula

$$
\left\langle\varepsilon^{2}\right\rangle=2 \kappa T^{2} \frac{d\langle E\rangle}{d T}
$$

from the framework developed in (Einstein, 1903) for a general statistical mechanics for systems of a finite number of degrees of freedom that obey arbitrarily given dynamical laws subject only to the constraint that energy is conserved. In that framework, $\kappa$ plays the role of a universal constant common to systems of whatever dynamical constitution.

It is fair to say that a major goal of (Einstein, 1904), if not the major goal, is the demonstration of (14) and its application to a special case of blackbody radiation. In the preceding section $(\S 3)$, Einstein had shown that in the "kinetic theory of atoms" the constant $\kappa$ has the physical significance of a proportionality factor between the absolute temperature and the mean kinetic energy per atom, thereby determining its numerical value to be $\kappa=\frac{1}{2} \frac{R}{N}$. The fluctuation formula provides a universal interpretation of the physical significance of $\kappa$, namely as the universal constant governing thermal stability of a system. Thus, if one has an independent handle on the scale of fluctuations of a known system, one could obtain an independent estimate of the numerical value of $\kappa$ and thus in turn of Avagodro's number N. Quoting Einstein himself:

The equation just found would permit an exact determination of the universal constant $\kappa$ if it were possible to determine the average value of the square of the energy fluctuation of a system; this, however, is not the case in the present state of our knowledge. There is only a single sort of physical system to which, we can at all surmise from experience, an energy fluctuation corresponds; this is an empty space filled with thermal radiation (Einstein, 1904, pp. 360-361).

This clearly establishes that it had not yet occurred to Einstein that, according to the kinetic molecular theory of heat, thermal fluctuations should produce observable displacements of microscopically visible particles suspended in a fluid. ${ }^{7}$

The type of system Einstein has in mind is a space filled with blackbody radiation. If the linear dimensions of the space are large in comparison to the wavelength $\lambda_{m}$ at which the radiation energy density is at its maximum, then, Einstein says, $\left\langle\varepsilon^{2}\right\rangle$ should be expected to be small in comparison with $\langle E\rangle^{2}$.

\footnotetext{
${ }^{7}$ Why, though, does Einstein say that there is only one sort of physical system for which we can surmise on the basis of experience the presence of energy fluctuations. In order to provide a contrast class for the explanation, ask why we cannot surmise the presence of energy fluctuations for rarified gases? In order to produce something parallel to what Einstein does for thermal radiation in the small, we would need at hand something to play the correlate of the Wien displacement law, according to which $\lambda_{m} T$ is a universal constant. But no such correlate appears to be available.
} 
On the other hand, if the size of the radiation space is of the order of magnitude of this wavelength, then the energy fluctuations should be expected to be of the same order of magnitude as the energy of the radiation in the space (Einstein, 1904, p. 361).

Einstein proceeds to explore the consequences of setting

$$
\left\langle\varepsilon^{2}\right\rangle=\langle E\rangle^{2}
$$

when the volume of the radiation space is

$$
V=\lambda_{m}^{3}
$$

According to the Stefan-Boltzmann law, the energy per unit volume of blackbody radiation is proportional to the fourth power of the absolute temperature $T$. Einstein takes this to remain valid for the average energy of a small space. Hence,

$$
\langle E\rangle=\sigma V T^{4},
$$

where $\sigma$ is the constant of proportionality of the Stefan-Boltzmann law. Substitution of this expression for $\langle E\rangle$ in the energy fluctuation formula (14) yields

$$
\left\langle\varepsilon^{2}\right\rangle=8 \kappa \sigma V T^{5} .
$$

From (15)-(18) it then follows that

$$
\lambda_{m} T=V^{1 / 3} T=2\left(\frac{\kappa}{\sigma}\right)^{1 / 3}=0.42,
$$

where Einstein uses the empirically established value $\sigma=7.06 \times 10^{-15}$ and the value $\kappa=6.6 \times 10^{-17}$ established in $\S 3$ from the kinetic theory of gases. Wien $(1893,1894)$ had established that for blackbody radiation $\lambda_{m} T$ is a universal constant. Measurements in the interim had established for it the value

$$
\lambda_{m} T=0.293
$$

The expression (19) agrees with (20), not only in functional form, but also, it seems, remarkably well numerically, especially given that the opening assumption involves an order of magnitude estimate. This, Einstein insists, should not be chalked up to chance. ${ }^{8}$

\footnotetext{
8 “. . . ich glaube, daß diese Übereinstimmung bei der großen Allgemeinheit unserer Voraussetzungen nicht dem Zufall zugeschrieben werden darf" (Einstein, 1904, p. 362).
} 


\section{$6 \quad$ Fluctuations in larger volumes}

The Stefan-Boltzmann law yields an expression for blackbody energy fluctuations at any scale. Let $V_{0}$ be a volume of blackbody radiation enclosed by perfectly reflecting mirrors and let $V$ be a fixed sub-volume of $V_{0}$. Then,

$$
\left\langle\varepsilon^{2}\right\rangle=8 \kappa \sigma V T^{5}=8 \kappa T\langle E\rangle,
$$

and

$$
\frac{\left\langle\varepsilon^{2}\right\rangle}{\langle E\rangle^{2}}=\frac{8 \kappa T}{\langle E\rangle}
$$

The kinetic theory of gases likewise yields simple results in the ideal case. Let $V_{0}$ be a volume of monatomic gas enclosed by perfectly reflecting walls and $V$ a fixed subvolume. The average energy per molecule of a monatomic gas is $3 \kappa T{ }^{9}$ If $\langle n\rangle$ is the average number of molecules ${ }^{10}$ in $V$, then the average energy in $V$ is $\left\langle E_{g}\right\rangle=3 \kappa\langle n\rangle T$. From the fluctuation formula (14),

$$
\left\langle\varepsilon_{g}^{2}\right\rangle=6\langle n\rangle \kappa^{2} T^{2}=3 \kappa T\left\langle E_{g}\right\rangle,
$$

and

$$
\frac{\left\langle\varepsilon_{g}^{2}\right\rangle}{\left\langle E_{g}\right\rangle^{2}}=\frac{3 \kappa T}{\left\langle E_{g}\right\rangle}=\frac{1}{\langle n\rangle} .
$$

Apart from the far right-hand side of the last, the two pairs of equations invite comparison. What does Maxwell's electromagnetic theory of light predict?

\subsection{Maxwell in the small}

Although he gives no rationale for it, Einstein's assertion, that $\left\langle\varepsilon^{2}\right\rangle \approx\langle E\rangle^{2}$ for blackbody radiation in a volume of linear dimension $\lambda_{m}$, is presumably based on the wave theory of light. The contemporary reader would have no reason to think otherwise. Presumably, the reasoning goes something like this. Since blackbody radiation is unpolarized, the behavior of the component of the electric field in any direction orthogonal to the $x$-direction, is as representative as any other. Choose, then, the $z$-component $Z$, and examine its instantaneous value $Z(t)$ at an arbitrarily given point. Each frequency $\nu$ contributes a term of the form

$$
Z_{\nu}=A_{\nu}(t) \sin 2 \pi \nu\left(t+\alpha_{\nu}\right)
$$

where $\left\langle A_{\nu}(t)\right\rangle \propto \sqrt{\varrho_{\nu}}$. For distinct frequencies $\nu$ and $\nu^{\prime}$, the phases $\alpha_{\nu}$ and $\alpha_{\nu^{\prime}}$, and the amplitudes $A_{\nu}(t)$ and $A_{\nu^{\prime}}(t)$ are related randomly. ${ }^{11}$ Because the blackbody spectrum

\footnotetext{
${ }^{9}$ If this sounds odd, keep in mind that, although Einstein's $\kappa$ plays the conceptual role of Boltzmann's constant, its numerical value is half that. Recall that (Einstein, 1904) demonstrates that its value, as determined by the kinetic theory of gases, is $R / 2 N$.

${ }^{10}$ If $n_{0}$ is the total number molecules in $V_{0}$, then $\langle n\rangle=\left(V / V_{0}\right) n_{0}$.

${ }^{11}$ Compare with Einstein's characterization of "maximally disordered" radiation in a footnote to $\S 1$ of the light-quantum paper (Einstein, 1905a, p. 153).
} 
(using either the Wien or the Planck distribution law) is sharply peaked around $\lambda_{m}$, the amplitudes for frequencies in the vicinity of $\lambda_{m}{ }^{-1} c$ strongly dominate. Thus, $Z(t)$ remains roughly periodic with mean period $\lambda_{m} c^{-1}$. But since the randomly varying phases of the $A_{\nu}$ 's close to the maximum of $\left\langle A_{\nu}\right\rangle$ sometimes conspire to greater constructive interference or greater destructive interference, $Z(t)$ displays significant fluctuations about $\langle Z(t)\rangle$. Figures 2 gives a rough ${ }^{12}$ illustration of both the amplitude and the period effects over a course of a five average-period stretch. Since the spatial variations in $Z(t)$ mirror the behavior of

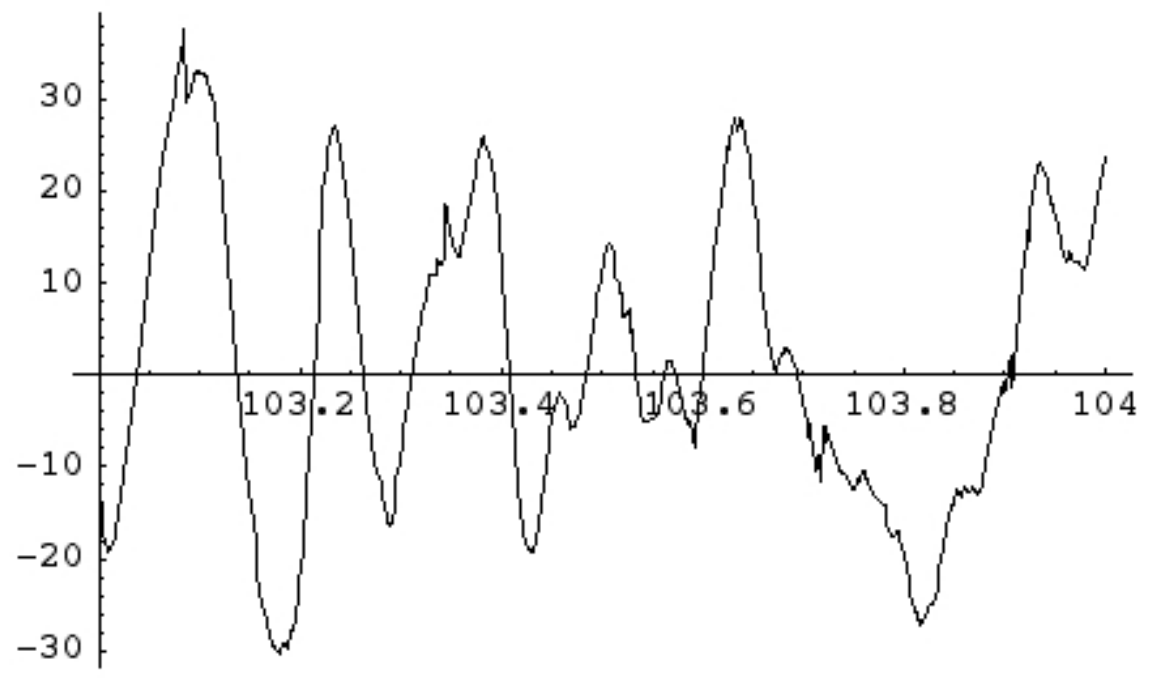

Figure 2: $Z(t)$ over 5 cycles with average period $=0.2$.

the temporal variations, it is easy to see that over sufficiently large volumes, of order of magnitude several times that of $\lambda_{m}{ }^{3}$, the energy fluctuations are small in comparison to average energy. However, at the scale of $\lambda_{m}$ they are quite significant.

How significant is a matter of guess. We have not been able to determine whether there are sufficiently tractable pencil and paper methods to get quantitative estimates. Our guess is that Einstein's estimate of $\left\langle\varepsilon^{2}\right\rangle \approx\langle E\rangle^{2}$, at least to the extent that it is based on Maxwell's theory, is uncanny guesswork based largely on visual intuition. ${ }^{13}$

\subsection{Maxwell in the large}

The very same physical intuitions that lead to the expectation of large energy fluctuations compared to total energy in the small permit one to see something about what Maxwell's

\footnotetext{
${ }^{12}$ For purposes of simplification, rather than pseudo-random number generators, we have used functions that do provide correlations between phases and amplitudes, but presumably correlations that are sufficiently pseudo-random in the Fourier series expansion.

${ }^{13}$ Our simulations were done, not surprisingly, by computer.
} 
theory predicts in the large.

Figure 3 illustrates the pattern over a time span sufficiently long to display coursegrained uniformity. Although the deviations of the energy density from the mean within

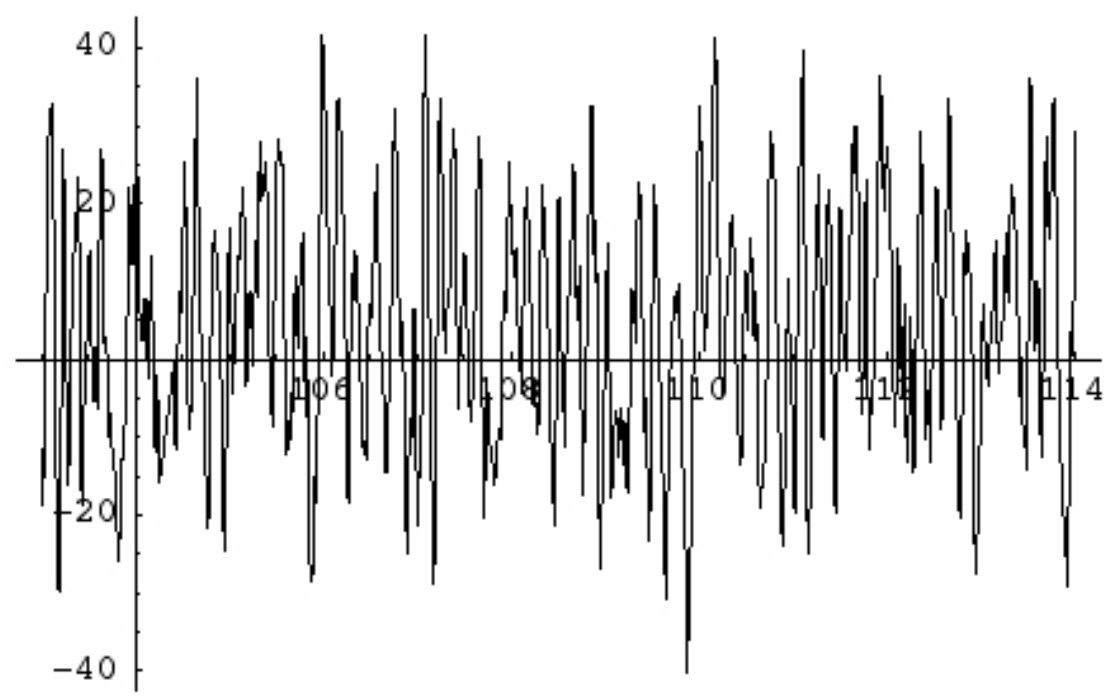

Figure 3: $Z(t)$ over 55 cycles with average period $=0.2$.

the subregions of a volume of size $\lambda_{m}{ }^{3}$ depend strongly on one another, if the volume is scaled up and the subregions are taken to be sufficiently large in comparison to $\lambda_{m}{ }^{3}$, then the deviations even in adjacent regions become statistically independent of one another, since the amount of energy to be partitioned along the border becomes insignificant in comparison to the total variations. This by itself, though, does not yield quantitative estimates.

In order to get these, first decompose the energy fluctuations into the fluctuations $\varepsilon_{\nu}$ corresponding to the different components of the radiation. Then, since, for a given spectral distribution $\varrho(\nu, T)$,

$$
\frac{d}{d T} \int_{0}^{\infty} \varrho(\nu, T) d \nu=\int_{0}^{\infty} \frac{d}{d T} \varrho(\nu, T) d \nu
$$

it follows in virtue of the fluctuation formula (14) that

$$
\left\langle\varepsilon^{2}\right\rangle=\int_{0}^{\infty}\left\langle\varepsilon_{\nu}^{2}\right\rangle d \nu
$$

Thus, the problem reduces to getting a handle on $\left\langle\varepsilon_{\nu}{ }^{2}\right\rangle$.

Here is where the physical intuitions about the scale of fluctuations in the small come back into play. Let $E_{\nu}^{P}$ be the instantaneous energy of a Planckian resonator with eigen- 
frequency $\nu$ and $\varepsilon_{\nu}^{P}$ its instantaneous energy fluctuation away from $\left\langle E_{\nu}^{P}\right\rangle$. Since the dimensions of the resonator are small in comparison to the wavelength corresponding to its eigenfrequency,

$$
\left\langle\left(\varepsilon_{\nu}^{P}\right)^{2}\right\rangle \approx\left\langle E_{\nu}^{P}\right\rangle^{2}
$$

Furthermore, Planck (1900) had established, independently of the functional form of $\varrho_{\nu}$, a relation we henceforth call Planck's relation:

$$
E_{\nu}^{P}=\frac{c^{3}}{8 \pi \nu^{2}} \varrho_{v}
$$

Hence, for a unit volume, the fluctuation formula (14) gives:

$$
\begin{aligned}
\left\langle\varepsilon_{\nu}{ }^{2}\right\rangle & =2 \kappa T^{2} \frac{d}{d T}\left\langle\varrho_{\nu}\right\rangle \\
& =2 \kappa T^{2} \frac{8 \pi \nu^{2}}{c^{3}} \frac{d}{d T}\left\langle E_{\nu}^{P}\right\rangle \\
& =\frac{8 \pi \nu^{2}}{c^{3}}\left\langle\left(\varepsilon_{\nu}^{P}\right)^{2}\right\rangle \\
& \approx \frac{8 \pi \nu^{2}}{c^{3}}\left\langle E_{\nu}^{P}\right\rangle^{2}
\end{aligned}
$$

Since it also follows from Planck's relation (28) that

$$
\left\langle E_{\nu}^{P}\right\rangle^{2}=\left(\frac{c^{3}}{8 \pi \nu^{2}}\right)^{2}\left\langle\varrho_{v}\right\rangle^{2}
$$

we obtain finally

$$
\left\langle\varepsilon_{\nu}{ }^{2}\right\rangle \approx \frac{c^{3}}{8 \pi \nu^{2}}\left\langle\varrho_{\nu}\right\rangle^{2}
$$

At this point, $\left\langle\varepsilon^{2}\right\rangle$ can be found by substituting a specific spectral distribution for $\varrho_{\nu}$ and integrating over all frequencies. Using Wien's simpler spectral law (2),

$$
\left\langle\varepsilon^{2}\right\rangle \approx 0.24 \kappa \sigma T^{5} \text {. }
$$

Comparison with (21) show that Maxwell's theory predicts that the mean square fluctuations are only about $3 \%$ of what is expected according to the Stefan-Boltzmann law. Using Planck's law raises the predicted amount by only a percentage point or two.

In the above calculations, the trustworthy value for $\langle\varepsilon\rangle$ was obtained by substituting the Stefan-Boltzmann formula, assumed to hold for average energies, into the fluctuation formula (14). Alternatively, since $\langle\varepsilon\rangle$ is the integral of $\left\langle\varepsilon_{\nu}{ }^{2}\right\rangle$ over all frequencies and $\left\langle E_{\nu}\right\rangle=$ $V \varrho_{\nu}$ is the total energy on average for radiation with frequency $\nu$, one can proceed by first calculating

$$
\left\langle\varepsilon_{\nu}^{2}\right\rangle=2 \kappa T^{2} V \frac{d \varrho_{\nu}}{d T}
$$


using a decently trustworthy expression for $\varrho_{\nu}$. Wien's spectral law (2), though not quite as accurate as Planck's, is far more tractable. The calculation yields

$$
\left\langle\varepsilon_{\nu}^{2}\right\rangle=2 \kappa V \beta \alpha \nu^{4} e^{-\beta \nu / T}=2 \kappa \beta \nu\left\langle E_{\nu}\right\rangle
$$

or,

$$
\frac{\left\langle\varepsilon_{\nu}^{2}\right\rangle}{\left\langle E_{\nu}\right\rangle^{2}}=\frac{2 \kappa \beta \nu}{\left\langle E_{\nu}\right\rangle}
$$

At a fixed temperature, this now invites comparison with equation (24) obtained for an ideal gas, viz.,

$$
\frac{\left\langle\varepsilon_{g}^{2}\right\rangle}{\left\langle E_{g}\right\rangle^{2}}=\frac{1}{\langle n\rangle},
$$

suggesting the interpretation

$$
\frac{\left\langle E_{\nu}\right\rangle}{2 \kappa \beta \nu} \sim\langle n\rangle .
$$

Since $2 \kappa$ is just $R / N$, this takes us full circle back to equation (13) from the end of our discussion of the entropic argument of the light-quantum paper.

If the analogy between equations (38) and (39) is less than compelling, then all the more reason to seek something like the entropic argument. Moreover, there is the issue as to how much weight the estimate that $\left\langle\left(\varepsilon_{\nu}^{P}\right)^{2}\right\rangle \approx\left\langle E_{\nu}^{P}\right\rangle^{2}$ can bear.

\section{The negative arguments of the light-quantum paper}

Remarkably, there is a simple consideration suggesting that (27) should hold, not just approximately, but as a strict equality. This is the equipartition theorem applied to resonators in dynamic equilibrium with a system of free electrons and molecules. Einstein lays this out in the course of the reductio argument in $\S 1$ of the 1905 light-quantum paper.

That reductio, as important as it is in its own right, also sets the stage for the argument in $\S 2$ of the light-quantum paper addressing the determination of the "elementary quanta of matter," in other words, Avogadro's number. This, it should not be forgotten, is the very note on which the 1904 paper ends. As Einstein wrote to his friend Conrad Habicht at the time:

I have now found in a most simple way the relation between the size of the elementary quanta of matter and the wavelengths of radiation (Einstein to Conrad Habicht, 15 April 1904, CPAE, Vol. 5, Doc. 18).

Explicitly, Einstein had shown that if $\left\langle\varepsilon^{2}\right\rangle \approx\langle E\rangle^{2}$ when $V \approx \lambda_{m}{ }^{3}$, then $\lambda_{m} T \approx 2(\kappa / \sigma)^{1 / 3}$ (cf. equation (19)). The kinetic theory of gases fixes $2 \kappa=R / N$. Thus, to the extent that the estimate of the sizes of energy fluctuations on the scale of $\lambda_{m}$ is reliable,

$$
N \approx \frac{4 R}{\sigma\left(\lambda_{m} T\right)^{3}}
$$


The two negative arguments of the light-quantum paper, therefore, make obvious contact with the concerns of the 1904 paper. Not so obvious is how completely intertwined they are.

\subsection{The Reductio}

In $\S 1$ of the light-quantum paper, Einstein asks us to consider a composite system consisting of three components bounded by completely reflecting walls. One is a collection of free molecules and free electrons colliding by conservative force interactions. A second is a number of electrons bound to fixed points by means of linear restoring forces (resonators). These are also allowed to interact with elements of the first component via conservative forces. According to Maxwell's theory, if these first two components are in dynamical equilibrium, the resonators must emit (and absorb) radiation (the third component) which has the same character as blackbody radiation if resonators of all frequencies are taken into consideration.

Now, and here is the crucial move, the average kinetic energy of an electron resonator, according to the kinetic theory of gases, must be the same as that of a free gas molecule. The equipartition theorem requires a resonator oscillating in a single dimension to have average energy

$$
\left\langle E^{P}\right\rangle=\frac{R}{N} T
$$

on average $(R / 2 N) T$ for the kinetic energy and $(R / 2 N) T$ for the potential energy, or $(R / 2 N) T$ for the two quadratic terms, $x^{2}$ and $p^{2}$, in the Hamiltonian. Recall, however, that according to Planck's relation

$$
\left\langle E_{\nu}^{P}\right\rangle=\frac{c^{3}}{8 \pi \nu^{2}} \varrho_{\nu}
$$

for each frequency $\nu$. Since (42) holds no matter what the frequency,

$$
\frac{R}{N} T=\frac{c^{3}}{8 \pi \nu^{2}} \varrho_{\nu}
$$

This completely determines $\varrho$ as a function of $\nu$ and $T$, namely,

$$
\varrho(\nu, T)=\frac{R}{N} \frac{8 \pi}{c^{3}} \nu^{2} T
$$

This spectral law, later known as the Rayleigh-Jeans law, is not only wildly at odds with experiment, but precludes the possibility of thermal equilibrium between ether and matter as the integral over available frequencies at fixed temperature grows without bound as the range of available frequencies grows. 
It is not out of place to ask what the Rayleigh-Jeans law (45) predicts for energy fluctuations. Substituting it into the frequency specific version of the fluctuation formula (36), we find for unit volume

$$
\left\langle\varepsilon_{\nu}{ }^{2}\right\rangle=4 \kappa^{2} T^{2} \frac{8 \pi \nu^{2}}{c^{3}}
$$

where we used that $2 \kappa=R / N$. The Rayleigh-Jeans law (45) can be rewritten as:

$$
T^{2}=\left(\frac{N}{R} \frac{c^{3}}{8 \pi \nu^{2}}\right)^{2} \varrho_{\nu}^{2}
$$

Substitution of this expression for $T^{2}$ in (46) results in the strict-equality version of (34):

$$
\left\langle\varepsilon_{\nu}^{2}\right\rangle=\frac{c^{3}}{8 \pi \nu^{2}}\left\langle\varrho_{\nu}\right\rangle^{2}
$$

This strict equality could have been achieved alternatively by invoking the kinetic theory of gases in the original derivation of (34). If $\left\langle E_{\nu}^{P}\right\rangle=2 \kappa T$, then, by the fluctuation formula (14),

$$
\left\langle\left(\varepsilon_{\nu}^{P}\right)^{2}\right\rangle=4 \kappa^{2} T^{2}=\left\langle E_{\nu}^{P}\right\rangle^{2}
$$

which can replace the estimate $\left\langle\left(\varepsilon_{\nu}^{P}\right)^{2}\right\rangle \approx\left\langle E_{\nu}^{P}\right\rangle^{2}$ based on the small scale fluctuations expected according to Maxwell's theory.

By digging into the background of Planck's derivation of a value for Avogadro's number $N$ in connection with his attempted justification of his newly discovered spectral law, we can gain some further insight into what might have inspired Einstein to bring the equipartition theorem of the kinetic theory into play.

\subsection{The Numbers Game}

As important as the reductio argument is for calling into question the received view about Maxwell's theory, it also has the subsidiary, and not insignificant function, of setting up a negative argument directed specifically at Planck.

In his work on blackbody radiation, Planck took as a major accomplishment, not just his spectral law,

$$
\varrho(\nu, T)=\frac{8 \pi h}{c^{3}} \nu^{3}\left(\frac{1}{e^{h \nu / k T}-1}\right),
$$

in which, Planck says, $h$ and $k$ play the role of "natural" constants, but also his proposal for independently determining from these a value for Avogadro's number and hence other fundamental constants such as the charge of the electron. It is of utmost importance here to understand that the constant $k$, later to become known as Boltzmann's constant, is not by definition set to $R / N$, but rather, like Planck's constant $h$, is a parameter in Planck's law that is determinable from other empirically measured constants governing the behavior 
of blackbody radiation, specifically the constants appearing in the Stefan-Boltzmann law and in Wien's displacement law, respectively. The details of this are sufficiently edifying to present them here.

The value of the universal constant $\lambda_{m} T$ is enough to determine the ratio of $h$ to $k$ in Planck's law. Just set to zero the derivative with respect to $\lambda$ of $\varrho$ expressed as a function of $\lambda$ and $T$, and take your best shot at numerically solving for $\delta:=c h / k \lambda_{m} T$ in the resulting transcendental equation ${ }^{14}$

$$
\left(1-\frac{\delta}{5}\right) e^{\delta}=1
$$

Then $^{15}$

$$
h=\delta k \frac{\lambda_{m} T}{c} .
$$

A second (independent) equation involving $h$ and $k$ results from the empirically determined value of the universal constant $\sigma$ in the Stefan-Boltzmann law. Integration of Planck's law (50) over all frequencies gives a total radiation energy $u(T)$ per unit volume at absolute temperature $T$ of

$$
u(T)=\frac{48 \pi k^{4}}{c^{3} h^{3}} \zeta(4) T^{4},
$$

where $\zeta(k)$ is Riemann's zeta function. ${ }^{16}$ Hence,

$$
\sigma=\frac{48 \pi k^{4}}{c^{3} h^{3}} \zeta(4)
$$

It follows, in virtue of $(51)$, that

$$
\begin{aligned}
k & =\frac{\delta^{3}}{48 \pi \zeta(4)}\left(\lambda_{m} T\right)^{3} \sigma \\
h & =\frac{\delta^{4}}{48 \pi \zeta(4)} \frac{\left(\lambda_{m} T\right)^{4} \sigma}{c},
\end{aligned}
$$

where $\delta^{3} / 48 \pi \zeta(4) \approx 0.75$ and $\delta^{4} / 48 \pi \zeta(4) \approx 3.72 .^{17}$

${ }^{14}$ More explicitly, Planck's law expressed in terms of $\lambda$ and $T$ reads

$$
\varrho(\lambda, T)=\frac{8 \pi h c}{\lambda^{5}}\left(\frac{1}{e^{h c / k \lambda T}-1}\right) .
$$

Setting $\partial \varrho / \partial \lambda=0$ yields

$$
\left(1-\frac{c h}{5 k \lambda_{m} T}\right) e^{c h / k \lambda_{m} T}=1 .
$$

${ }^{15}$ Planck (1901a) uses the approximation $\delta=4.9651$.

${ }^{16}$ Riemann's zeta function is given by $\zeta(n)=\sum_{k=1}^{\infty} k^{-n}$. For $n=4$, Planck uses the numerical approximation $\zeta(4)=1.0823$ rather than the exact solution $\zeta(4)=\pi^{4} / 90$.

${ }^{17}$ Wien's spectral law,

$$
\varrho(\nu, T)=\frac{8 \pi h}{c^{3}} e^{-h \nu / k T}
$$


Compare the expression (53) for $k$ above with the expression

$$
2 \kappa \approx \frac{1}{4}\left(\lambda_{m} T\right)^{3} \sigma
$$

implicit in Einstein's 1904 paper. There $\kappa$ has the status of a universal constant governing all thermal systems. The fluctuation argument is intended to show that $\kappa$ stands in this functional relation to the constants $\lambda_{m} T$ and $\sigma$ governing the characteristics of thermal radiation. To the extent that he is satisfied that the derived value for $\lambda_{m} T$ is tolerably accurate, he is satisfied, in the first instance, that Planck's $k$ equals $2 \kappa$, and secondly, since he has established the relation between $\kappa$ and $N$ for the kinetic theory of gases, that Planck's $k$ equals $R / N$.

Planck's (1901a) derivation of Planck's law, however, forges no such connection between $k$ and Avogadro's number. In that derivation, $h$ and $k$ are introduced as arbitrary theoretical parameters whose values are subsequently evaluated from the empirically known values of $\sigma$ and $\lambda_{m} T$. The pair $h$ and $k$ serve only to provide a change of basis, so to speak, from the pair $\sigma$ and $\lambda_{m} T$ for the two independent parameters in Planck's model. They are universal constants only for the domain of blackbody radiation.

In particular, (Planck, 1901a) introduces $k$ as a proportionality constant in a proposed definition of electromagnetic entropy for electron resonators. Suppose that the total energy $U_{n}$ for a system of $n$ identical resonators is divided into $P$ "energy elements" of size $\varepsilon$ distributed over the $n$ resonators. ${ }^{18}$ Let $\mathfrak{R}$ be the number of distinct ways that these $P$ elements can be so distributed. The entropy $S_{n}$ of the system is defined, up to an additive constant, as

$$
S_{n}={ }_{d f} k \log \mathfrak{R} .
$$

Planck mentions that the definition recommends itself for its simplicity and its close "kinship" with a result from the kinetic theory of gases. ${ }^{19}$ However, a little more than a year earlier, he had introduced a different definition of electromagnetic entropy in order to derive Wien's spectral law, which, at the time, he thought commanded strict empirical validity (Planck, 1900). He went so far as to claim that this definition, as well as Wien's spectral law, are necessary for the second law of thermodynamics to hold for the electromagnetic theory of light. ${ }^{20,21}$ The true modus operandi - choose a definition of electromagnetic energy that yields the desired spectral law - could hardly be missed.

\footnotetext{
yields the simpler expressions $k=\left(5^{3} / 48 \pi\right)\left(\lambda_{m} T\right)^{3} \sigma$ and $h=\left(5^{4} / 48 \pi\right)\left(\lambda_{m} T\right)^{4} \sigma$.

${ }^{18}$ That $\varepsilon=h \nu$ for the characteristic frequency $\nu$ of the resonators is subsequently derived.

19 "Für die Zweckmässigkeit der so getroffenen Festsetzung lässt sich von vornherein ihre Einfachheit und ihre nahe Verwandtschaft mit einem Satze der kinetischen Gastheorire anführen" (Planck, 1901a, p. 556). The result Planck has in mind is the one from (Boltzmann, 1877) that Planck introduces explicitly in (Planck, 1901b).

20 "Ich glaube hieraus schliessen zu müssen, dass die im $\S 17$ gegebene Definition der Strahlungsentropie und damit auch das Wien'sche Energiervertheilungsgesetz eine notwendige Folge der Anwendung des Principes der Vermehrung der Entropie auf die elektromagnetische Strahlungstheorie ist ..." (Planck, 1900, p. 118).

${ }^{21}$ See (Gearhart, 2002) for more on Planck.
} 
Planck (1901b), which is essentially an addendum to (Planck, 1901a), attempts to link $k$ to the constants appearing in the kinetic theory of gases. He first extracts a result from (Boltzmann, 1877), that the entropy for a monatomic gas in thermal equilibrium is

$$
\int \frac{d Q}{T}=\frac{R}{N} \log \mathfrak{P}
$$

where $\log \mathfrak{P}$ is the "natural logarithm of the probability of the stationary velocity distribution among the atoms, as measured by the number $\mathfrak{P}$ of possible "complexions'." ${ }^{22} \mathrm{He}$ then gives the following argument for the identity of the coefficients in the two expressions (56) and (57) for entropy. Suppose that, in addition to the gas molecules, radiating resonators are present. Then the total number of complexions of the combined system is $\mathfrak{P} \cdot \mathfrak{R}$ "since, according to the electromagnetic theory of radiation, the velocities of the atoms are completely independent of the distribution of the energy radiated." 23 Thus, for some proportionality constant $f$,

$$
f \log (\mathfrak{P} \cdot \mathfrak{R})=f \log \mathfrak{P}+f \log \mathfrak{R} .
$$

From the expressions (56) and (57) for the gas and the resonator entropies, respectively, it follows that $f=k=R / N$.

It is hardly a reach to see Einstein's derivation of "Boltzmann's principle" in $\S 5$ of the light-quantum paper as a generalization of this argument to arbitrary systems. Ironically, though, there are legitimate doubts whether Planck's argument is in fact a valid instance of Einstein's generalization, doubts of the sort that serve to set up Einstein's reductio argument from $\S 1$. It may be granted that the thermal radiation does not interact with the gas molecules. But Planck's argument tacitly assumes that the gas molecules and the resonators do not interact either. And if the system of resonators and radiation is dynamically isolated from the gas, they need not share the same temperature. A link between electromagnetic temperature and temperature as defined in the kinetic theory can be forged only through composite systems in dynamical interaction. Ergo the Gedanken system that sets up the reductio argument. The introduction of free electrons over and above the gas molecules removes any doubt concerning dynamical interaction with the resonators. And, according to the electron theory of metals, the electrons can be treated according to the principles of the kinetic theory in the same way as a gas. ${ }^{24}$ This ensures

\footnotetext{
22 “. . $\log \mathfrak{P}$ [bedeutet] den natürlichen Logarithmus der durch die Anzahl $\mathfrak{P}$ der möglichen 'Complexionen' gemessenen Wahrscheinlichkeit der stationären Geschwindigkeitsverteilung unter den Atomen” (Planck, 1901b, p. 564).

23 "[d]a aber nach der elektromagnetischen Theorie der Strahlung die Geschwindigkeiten der Atome vollkommen unabhängig sind von der Verteilung der strahlenden energie, ..." (Planck, 1901b, p. 565).

${ }^{24} \mathrm{Cf}$. the footnote (Einstein, 1901a, p. 133): "This assumption [that the mix of electrons and molecules can collide with one another in the same way as gas molecules according to the kinetic theory of gases] is equivalent to the postulate that the average kinetic energy of the electrons are equal to one another in thermal equilibrium. With the help of the latter postulate, Hr. Drude, as is well known, has derived the ratio of thermal to electrical conductivity in metals on theoretical grounds."
} 
the crucial application of the equipartition theorem to the resonators as well, the move on which the whole reductio rests.

Now back to the professed purpose of $\S 2$ of the light-quantum paper, namely, that Planck's theory of blackbody radiation is completely unnecessary for the determination of Avogadro's number. Planck's law taken as an empirical generalization suffices. In Einstein's notation, the first few terms in the series expansions of Planck's law in $(\nu / T)$ are

$$
\frac{\alpha \nu^{3}}{e^{\beta \nu / T}-1}=\frac{\alpha}{\beta} \nu^{2} T\left\{1-\frac{\beta}{2}\left(\frac{\nu}{T}\right)+\frac{\beta^{2}}{12}\left(\frac{\nu}{T}\right)^{2}-\cdots\right\}
$$

Thus, as $(T / \nu)$ grows sufficiently large,${ }^{25}$ Planck's law goes over to

$$
\varrho_{\nu}=\frac{\alpha}{\beta} \nu^{2} T
$$

which has exactly the same form as the Rayleigh-Jeans law (45). Comparison of the coefficients of $\nu^{2} T$ in the two cases yields

$$
N=\frac{8 \pi \beta R}{\alpha c^{3}}
$$

wherein all the constants on the right-hand side can be determined experimentally. Einstein concludes that the theoretical foundations that lead to the Rayleigh-Jeans law (45) are trustworthy if (but of course only if) the wavelengths are sufficiently long and the temperature sufficiently high.

The argument suffers from a minor lacuna. That Planck's law converges to the same form as the Rayleigh-Jeans in the limit of large $(T / \nu)$ certainly suggests that they agree in the classical limit, but it does not rigorously entail this without the value of $N$ already being known. And equation (60) tells us the value of $N$ only if it is assumed that Planck's law has the right classical limit. This is a serious issue. We know that at some stage of his work on blackbody radiation and the quantum hypothesis it dawned on Einstein that Planck's law is strictly correct in the classical limit. But when and how? If it was not just a leap of faith, the following deserves a fair hearing.

Just as Wien's spectral law can be inserted into the frequency-specific form of the energy-fluctuation formula (36) in order to obtain an approximate result for $\left\langle\varepsilon_{\nu}\right\rangle$, Planck's law can be used to obtain a more adequate result. At the first stage of calculation one obtains for unit volume

$$
\left\langle\varepsilon_{\nu}\right\rangle=2 \kappa \alpha \beta \nu^{4} \frac{e^{\beta \nu / T}}{\left(e^{\beta \nu / T}-1\right)^{2}},
$$

which doesn't appear to be very illuminating. But this can be rewritten

$$
\left\langle\varepsilon_{\nu}\right\rangle=\frac{2 \kappa \alpha \beta \nu^{4}\left(e^{\beta \nu / T}-1\right)}{\left(e^{\beta \nu / T}-1\right)^{2}}+\frac{2 \kappa \alpha \beta \nu^{4}}{\left(e^{\beta \nu / T}-1\right)^{2}}
$$

\footnotetext{
${ }^{25}$ I.e., when $\beta \nu / T \ll 1$.
} 


$$
=2 \kappa \beta \nu \varrho_{\nu}+\frac{2 \kappa \beta}{\alpha \nu^{2}} \varrho_{\nu}^{2} .
$$

The first term on the right-hand side is just the result obtained on the basis of Wien's spectral law (cf. eq. (37)). Since, according to Einstein's transcription of Planck's law,

$$
\alpha=\frac{8 \pi h}{c^{3}}
$$

and

$$
\beta=h / k,
$$

the second term on the right-hand side of (63) reduces to

$$
\frac{2 \kappa}{k} \frac{c^{3}}{8 \pi \nu^{2}} \varrho_{\nu}^{2}
$$

If $2 \kappa=k$, then Planck's law also yields the energy fluctuation term expected from Maxwell's equations. Although this is still not a proof that Planck's law contains the classical limit, the accumulation of coincidences becomes overwhelmingly convincing.

\section{Moving mirrors}

Our principal suggestion has been that Einstein's realization that Maxwell's theory fails to account for the bulk of the energy fluctuations that should be present for blackbody radiation represents a pivotal moment leading to the light-quantum paper. We would also like to point to how it may have played an important role in the genesis of his other two great works of the annus mirabilis.

\subsection{Brownian motion}

If Maxwell's theory cannot account for the energy fluctuations, then, as an immediate corollary, neither can it account for the expected pressure fluctuations.

When Max von Laue asked Einstein for comments on a relativity text he had written, Einstein replied:

If one goes through your inventory of confirmations of the special theory of relativity, one would think that Maxwell's theory is to be regarded as secure. However by 1905 I already knew for certain that it led to false fluctuations in radiation pressure and thereby to an incorrect Brownian motion of a mirror in a Planckian radiation cavity ${ }^{26}$ (Einstein to Max von Laue, 17 January 1952, Document EA 16-167, Einstein Archives).

\footnotetext{
${ }^{26}$ Wenn man Deine Kollektion der Bestätigungen der speziell. Rel. Theorie durchgeht, so meint man, die Maxwell'sche Theorie sei zum Greifen sicher. Aber 1905 wusste ich shon sicher, dass sie zu falschen Schwankungendes Strahlungsdruckes führt und damit zu einer unrichtigen Brown'schen Bewegung eines Spiegels in einem Planck'schen Strahlungs-Hohlraum.
} 
How could one not be tempted to explore at what scale this effect might be observable? Not only would it serve as direct confirmation of the inadequacy of Maxwell's theory, but it would yield an independent estimate of the universal constant $\kappa$ and, as a byproduct, Avogadro's number $N$.

If a fully reflecting mirror of finite thickness is suspended in a radiation cavity and free to move normal to its surface, then the radiation pressure on the two sides will in general be different due to fluctuations in radiation energy, thus setting the mirror in motion. Once in motion, the radiation pressure acts as a frictional force tending to bring the mirror to rest. If the kinetic theory is applicable, the kinetic energy of the mirror will fluctuate about a mean value of $(1 / 2) k T$, and the mirror will execute a one-dimensional random walk about its initial position.

The problem is formally analogous to calculating the displacements of a rigid body suspended in a viscous fluid and subject to pressure fluctuations. In early 1903, Einstein had entertained the problem of a rigid sphere, the size of a large molecule, moving in a viscous fluid under the influence of a potential gradient, as a way of calculating Avogadro's number, a project that ultimately resulted in his doctoral dissertation (Einstein, 1905b), finished April 30 of 1905. In a letter dated March 17, he asks Michele Besso:

Have you already calculated the absolute size of ions on the assumption that these are spheres and are large enough that the equation of the hydrodynamics of viscous fluids are applicable[?] Given that we know the absolute charge of the electron, this should be quite an easy matter. I would have done it myself, but I lack the literature and time. You could also take advantage of diffusion in order to obtain results with neutral salt molecules in solution. If you don't get what I mean, I'll gladly write to you with further details (Einstein to Michele Besso, 17 March 1903, CPAE, Vol. 5, Doc. 7).

The dissertation takes up the second model using sugar molecules for the solute. Osmotic pressure plays the role of the potential difference, inducing an applied force $K$ on each molecule which drives it through the fluid at a constant velocity

$$
v=\frac{K}{6 \pi r \chi}
$$

inversely proportional to the coefficient of friction $\chi$ and the radius $r$ of the molecule. This yields an expression for the diffusion coefficient,

$$
D=\frac{R}{N} \frac{T}{6 \pi r \chi}
$$

where $T$ is the absolute temperature. (A second equation relating $N$ and $r$ from a calculation of the change in viscosity due to the introduction of the solute - a calculation which takes up the bulk of the dissertation - yields numerical solutions for $N$ and $r$, respectively.) 
This model adapts itself to the case of the moving mirror by substituting radiation pressure for osmotic pressure. An analogue of equation (67) results from using surface area instead of radius and taking $\chi$ to be the coefficient of friction of radiation pressure. The expression (68) for the diffusion constant is obtained with the help of Van 't Hoff's law for osmotic pressure,

$$
p=\frac{R}{N} \frac{\mu}{m} T
$$

where $\mu$ is the mass per unit volume and $m$ the molecular weight of the solvent. Here the analogy breaks down, since the osmotic pressure is a (linear) function of the number of moles of solute introduced, whereas the radiation pressure is not a function of any properties of the mirror introduced. Thus, there is no obvious way of getting an analogue of the diffusion constant. Nonetheless, dwelling on the role of a diffusion constant in the two cases brings to light an unexpected feature common to the two problems.

The kinetic theory from the beginning was known to predict that fluctuations must exist on at least molecular scales; according to conventional wisdom, fluctuations on larger scales would remain unobservable. The mass of a sufficiently large body would make negligible the velocity corresponding to a mean kinetic energy equal to $(3 / 2) k T$. In the case of the mirror, though, it is really the surface area, and not the mass per se, that governs the scale of the displacements in the expected random walk. On reflection, something similar should hold for a sphere suspended in a viscous fluid subject to fluctuations in osmotic pressure, and indeed, it is the radius of the body, not its mass, that appears in the expression (68) for the diffusion constant $D$. If this expression is used as the constant of proportionality in the diffusion equation

$$
\frac{\partial f}{\partial t}=D \frac{\partial^{2} f}{\partial x^{2}}
$$

microscopically observable random walks are predicted for particles with radii on the order of $10^{-3} \mathrm{~mm}$, as predicted in the Brownian motion paper. All that remains to be established is that, according to the "general molecular theory of heat," such particles in suspension do indeed give rise to an osmotic pressure in the same way as chemical substances in solution do.

\subsection{Relativity}

The existence of radiation pressure can be established on either purely thermodynamical grounds or on the basis of Maxwell's theory. Even if Maxwell's theory gives the wrong predictions for radiation fluctuations, it can still be relied on for time-average values. The introduction of the light quantum has the virtue of making up for the deficit in the energy fluctuations of blackbody radiation. It suggests, however, a very different picture of the mechanism producing radiation pressure. Does it give the right quantitative results?

For a monochromatic ray incident at an angle $\theta$ to the normal of the surface of a completely reflecting mirror with velocity $v$, the problem turns out to be simpler than if 
treated as a problem in wave optics where the angle of reflection, which, due to aberration, in general differs from $\theta$, needs to be taken into account. The problem becomes especially simple if the rest frame of the mirror is treated as the "stationary" frame, a move Einstein had come to regard as legitimate well prior to 1905. All that matters then in the lightquantum case is the net gain or loss in energy due the Doppler shift in frequency upon reflection. This sort of calculation invites itself, not just as an interesting exercise, but as an essential test of the consistency of the light-quantum heuristic with Einstein's views developed to date on he electrodynamics of moving bodies.

As is well known, Einstein had talked himself into the principle of relativity by convincing himself that, in order to restore causal symmetry to the explanation of the production of an electric current in electromagnetic induction, the electric field is a frame relative quantity. ${ }^{27}$ But if the fundamental equations of electrodynamics are to abide by the principle of relativity, it would seem that, unlike in Maxwell's theory, the velocity of light (even in the "stationary" frame) must depend on the velocity of the source. Einstein struggled for a number of years to develop such an "emission" theory, but without success. Despite the lack of any direct experimental evidence one way or the other, he eventually convinced himself that, despite their apparent non-comformity with the principle of relativity, the Maxwell-Lorentz equations (in the form they have for the "stationary" frame) should, in any inertial frame, be an adequate description of optical phenomena involving the timeaverage field quantities, ${ }^{28}$ in particular, insofar as they characterize the velocity of light as independent of the motion of the source, a feature Einstein subsequently raised to the status of a postulate in the special theory of relativity.

Moreover, Einstein had developed this conviction by the time he wrote up the lightquantum heuristic for publication. Two passages from the light-quantum paper suffice to establish this. In the introduction, he states:

The wave theory of light [in particular, Maxwell's theory], which operates with spatially continuous [field] functions, has proved itself admirably for the representation of purely optical phenomena and will probably never be replaced by another $^{29}$ (Einstein, 1905a, p. 133).

In $\S 3$, he begins the derivation that the partial derivative of entropy density with respect

\footnotetext{
${ }^{27}$ Magneto-electric induction suggests the same for the magnetic field.

${ }^{28} \mathrm{Also}$, the fields should not be too strong, i.e., of the order of magnitude of the electric field of an electron in a region of its classical radius. Einstein recognized that Maxwell's equations would not suffice here, else there is no accounting for the stability of the electron under its own electrostatic repulsion.

${ }^{29}$ That is, insofar as it deals with time-average field quantities. For the quote continues:
}

However, it is to be kept in mind that observations in optics concern time average values, not instantaneous values, and it is perfectly conceivable that, despite the complete experimental confirmation of the theory of diffraction, reflection, refraction, dispersion, etc., that the theory of light using spatially continuous functions leads to contradiction with experiment if applied to phenomena of light production and light transformation (Einstein, 1905a, pp. 132-133). 
to energy density is equal to $1 / T$ with the assumption that "the observable properties of the radiation at hand are completely determined if the radiation density $\varrho(\nu)$ is given for all frequencies" (Einstein, 1905a, p. 137). A footnote to this alludes to the lack of direct experimental evidence against an emission theory:

This assumption is an arbitrary one. One will naturally stay with the simplest assumption as long as experiment does not force one to reject it (Einstein, 1905a, p. 133).

Given that Einstein recognized the truly iconoclastic character of the light-quantum heuristic, it would be remarkable had he rushed into print without first investigating the consistency of the heuristic with the principle of relativity and the principle that the speed of light is source independent.

The "Electrodynamical Part" of the 1905 relativity paper contains two sections concluding with a pair of results bearing on just this point. One of them is the calculation of the relativistic expression for the pressure of radiation on a moving mirror. We saw above why this is important for the light-quantum heuristic. The other result is that the energy (per unit volume) and frequency of light transform according to the same formula, a result that Einstein singles out as noteworthy. This is necessary if the number of light quanta per unit volume is to be frame invariant. Otherwise the heuristic is as good as dead. One of us has explored in detail how these calculations proceed on the light-quantum picture (Rynasiewicz, 2005). To complete them, one needs also to arrive at the Lorentz transformations. But we see no reason why Einstein could not have been in possession of these prior to the discovery of the relativity of simultaneity. Consistency of the light-quantum picture with the principle of relativity and the principle of the constancy of light does not require an understanding of the physical grounds for the validity of the Lorentz transformations. It suffices for Einstein to have convinced himself that, whatever the laws of the fundamental theory yet to be discovered, everything happens as if the Lorentz transformations are correct, much in the same way as everything happens as if rigid bodies undergo a Lorentz contraction as a result of their motion through the ether.

\section{The birth of the light quantum}

Although we have emphasized the role of radiation fluctuations in the development of the light-quantum heuristic and suggested how this may have led to important insights for the other two papers of the annus mirabilis, we do not want to suggest that the very idea of the light quantum was born only after the discovery that Maxwell's theory fails miserably for energy fluctuations. 


\subsection{Relative Chronology}

As noted earlier, the rationale behind the assertion in (Einstein, 1904) that, in a volume of the order of magnitude $\lambda_{m}{ }^{3}$, the energy fluctuations of blackbody radiation should be roughly on the order of the mean energy, presumably derives from an examination of wave superposition at that scale in accordance with Maxwell's theory of light. If so, does it not follow that Einstein was still working within the framework of Maxwell's theory and had not yet conceived of the light quantum? Not if the assumption is justified on the light-quantum picture, as well. For then, Maxwell's theory, although sufficient, would not be necessary for the assumption. It does not matter for Einstein's purposes if the unsuspecting reader does not also recognize that Maxwell's theory is unnecessary.

Suppose it had occurred to Einstein by early 1904 that radiation consists of independent energy quanta $\epsilon(\nu)=2 \kappa \beta \nu$ in the Wien regime. Then

$$
\int_{0}^{\infty} \frac{\alpha \nu^{3} e^{-\beta \nu / T}}{\epsilon(\nu)} d \nu=\frac{\alpha}{\kappa \beta^{4}} T^{3}
$$

is the total number of quanta (for all frequencies) per unit volume. Since,

$$
\int_{0}^{\infty} \alpha \nu^{3} e^{-\beta \nu / T} d \nu=6 \frac{\alpha}{\beta^{4}} T^{4}
$$

is the total energy per unit volume, the average energy per light quantum is

$$
\bar{\epsilon}=6 \kappa T .
$$

Thus, according to the Stefan-Boltzmann law, there are on average

$$
\langle n\rangle=\frac{\langle E\rangle}{\bar{\epsilon}}=\frac{\sigma}{6 \kappa} V T^{3}
$$

light quanta in a given volume $V$. For a volume of linear dimension $\lambda_{m}$,

$$
\langle n\rangle=\frac{\langle E\rangle}{\bar{\epsilon}}=\frac{\sigma}{6 \kappa}\left(\lambda_{m} T\right)^{3} .
$$

Hence, given the empirically determined value $\lambda_{m} T=0.293$, there are $\langle n\rangle=0.46$ quanta in such a space - telling enough. From (73), the mean energy is $\langle E\rangle=2.76 \kappa T$. Applying the energy fluctuation formula (14), the mean square energy fluctuation is

$$
\left\langle\varepsilon^{2}\right\rangle=5.52 \kappa T^{2}=0.72\langle E\rangle^{2} .
$$

Thus, on either the wave or the light-quantum picture, the predicted fluctuations are on the order of the average total energy. Not only is it possible that the light quantum occurred to Einstein prior to (Einstein, 1904), we urge that it is more likely than not.

We have seen already the extent to which $\S 1$ and $\S 2$ of the light-quantum paper harbor critical attacks against Planck. Overtones of the same are unmistakable in (Einstein, 1904). 
First, an expression for the entropy of a system will be derived which is completely analogous to the one established by Boltzmann for ideal gases and assumed by Planck in his theory of radiation. ... Then the significance of a universal constant, which plays an important role in the general molecular theory of heat, will be sought. Finally, there follows an application to the theory of the radiation of black bodies, from which emerges, without the help of special hypotheses, a most interesting relation between the just mentioned universal constant, determined by the sizes of the elementary quanta of matter and electricity, and the order of magnitude of radiation wavelengths (Einstein, 1904, p. 354).

The phrase occurring in the last paragraph, "the elementary quanta of matter and electricity," is taken directly from the title of (Planck, 1901b): "Ueber die Elementarquanta der Materie und der Elektricität." To a significant extent, the thrust of (Einstein, 1904) is to show, given perceived but unmentioned deficiencies in Planck's approach, the superiority of Einstein's "general molecular theory of heat" in achieving the same ends. The expression for entropy to be derived is one established [gefunden] by Boltzmann in the case of ideal gases, but assumed [vorausgesetzt] by Planck in his theory of radiation - an unmistakable contrast and implicit criticism. The relation in the final section will be obtained without the help of special hypotheses - again, in contrast to Planck.

Years later, Besso mused to Einstein in a letter dated January 17, 1928:

As far as I'm concerned, I was your audience in the years 1904 and 1905; if, in the drafting of your publications on the quantum problem, I deprived you of some of your glory, in exchange I provided you with a friend in Planck (Einstein and Besso, 1972, p. 238).

The letter intimates that, at least from Besso's perspective as an insider, (Einstein, 1904) is to be counted among Einstein's "publications on the quantum problem." It also suggests that Einstein may have had more in his arsenal in 1904 than he let on, in particular, the reductio argument and the attendant alternative method of obtaining Avogadro's number by taking Planck's law to be correct in the classical limit. In (Einstein, 1905a), they set the stage for the positive heuristic to be articulated. If voiced in (Einstein, 1904), that paper would have read primarily as an assault on Planck with little in the way of a positive alternative to advance.

\subsection{The possibility of "double discovery"}

If the idea of the light quantum had occurred to Einstein before (Einstein, 1904), when did it occur to him and what suggested it?

Einstein's first publication on the light-quantum heuristic after (Einstein, 1905a) contains a revealing comment in the introduction: 
At that time [i.e., in 1905], it seemed to me as though Planck's theory of radiation stood in a certain sense in opposition to my work (Einstein, 1906b, p. 199).

In what sense?

The thrust of the 1906 paper is to show that Planck's law can be obtained if one explicitly quantizes the energy levels of Planckian resonators as the mechanism for energy exchange between radiation and matter in blackbody radiation. If one understands this to have been Planck's proposal, then what might be taken to have been valid criticisms of Planck's theory no longer have force. Rather, Planck is to be credited with having introduced into physics "a new hypothetical element - the light-quantum hypothesis" (Einstein, 1906b, p. 203).

Significant for our concerns is not the ruse to invite Planck to accept the light-quantum heuristic by offering him credit for its invention, but that there is no evidence that prior to this Einstein had considered quantizing the energy levels of material systems. Despite recognizing Planck's law, rather than Wien's spectral law, as generally empirically valid, (Einstein, 1905a) gives no hint as to how it might be derived from more fundamental principles. It does not even raise the concern. Planckian resonators appear in the context of the reductio argument, and, given that it is a reductio, the obvious assumption to reject is that, for blackbody radiation, the energy exchange between radiation and matter is mediated by Planckian resonators obeying Planck's relation (28) based on Maxwell's theory. After that, resonators make no further appearance. Before launching the positive entropic argument, Einstein announces, "in the following, blackbody radiation shall be considered in connection with experience without laying the groundwork for a picture concerning the production and propagation of radiation" (Einstein, 1905a, p. 137). However, the three sections addressing experimental consequences of the light-quantum heuristic do introduce pictures for the mechanism of the production and transformation of light.

For the process of photoluminescence, what is to be explained is why, according to Stokes' rule, the emitted frequencies are lower than the incident frequencies.

The transformation process is to be thought of as follows. Each incident energy quantum of the frequency $\nu_{1}$ is absorbed and by itself - at least if the distribution energy of the incident energy quantum is sufficiently small — gives rise to the production of a light quantum of the frequency $\nu_{2}$; frequently when the incident light quantum is absorbed also light quanta of the frequencies $\nu_{3}$ and $\nu_{4}$ can arise, as well as the production of other forms of energy (e.g., heat) (Einstein, 1905a, p. 144).

In the photoelectric effect, the production of cathode rays is triggered as follows.

According to the conception that the incident light consists of energy quanta of the energy $(R / N) \beta \nu$, the production of cathode rays by light can be understood 
in the following way. Energy quanta penetrate into the surface layer of the body, and their energy is converted, at least in part, into the kinetic energy of electrons. The simplest picture is that one light quantum surrenders is entire energy to a single electron (Einstein, 1905a, p. 145).

In the final application:

We will have to assume that when a gas is ionized by ultraviolet light a single absorbed light energy quantum is used to ionize a single gas molecule (Einstein, 1905a, pp. 147-148).

In none of these cases is there the slightest hint that the mechanisms are governed by the then accepted laws of electrodynamics. Indeed, given that they involve light quanta, it is hard to see how they could. Whatever the details of the mediating processes, they are, as Einstein says, irrelevant. ${ }^{30}$ All proceeds as though there is a direct conversion between radiant and other forms of energy.

In late April of 1901 Einstein wrote to Mileva Marić:

Recently the idea came to me that in the production of light perhaps a direct conversion of the energy of motion into light occurs on account of the parallel: kinetic energy of molecules - absolute temperature - spectrum (spatial energy of radiation in equilibrium) (Einstein to Mileva Marić, 30 April 1901, CPAE, Vol. 1, Doc. 102).

The first term in the parallel, the kinetic energy of molecules, may have been an allusion to Maxwell's velocity distribution for ideal gas molecules,

$$
d n_{v}=4 n \sqrt{\frac{\mathfrak{h}^{3} m^{3}}{\pi}} v^{2} e^{-\mathfrak{h} m v^{2}} d v
$$

where $d n_{v}$ is the number of molecules, treated as a continuous quantity, with velocity lying between $v$ and $v+d v$ out of the total number $n$. The quantity $\mathfrak{h}$ is the inverse of the temperature scaled into energy units by $2(R / N)$, i.e.,

$$
\mathfrak{h}=\frac{1}{2(R / N) T} .
$$

For there is a degree of formal similarity between this and Wien's spectral law (2), particularly as regards the exponential terms in the respective equations. In any event the exponent in Maxwell's distribution is $(N / R) T^{-1}$ times the kinetic energy of a molecule with velocity $v$, suggesting a parallel via the absolute temperature $T$ between molecular

\footnotetext{
30 "Unter Vermittelung von was für Zwischenprozessen dies Endresultat zustande kommt, ist gleichgültig" (Einstein, 1905a, p. 144).
} 
kinetic energy and $(R / N) \beta \nu$. If this is a quantum of energy to be associated with radiation of frequency $\nu$, then, as we saw from equations (71) and (72), ${ }^{31}$ the mean energy of a quantum of radiation is $3(R / N) T$, in striking parallel with $(3 / 2)(R / N) T$ for the average kinetic energy of a gas molecule.

Later the next month, Einstein begins a letter to Mileva Marić, not with the usual doting of lovers, but:

I just read a marvelous article by Lenard on the production of cathode rays by ultraviolet light. Under the influence of this beautiful piece I am filled with so much joy and so much delight that you must absolutely have some of it, too (Einstein to Mileva Marić, 28 May 1901, CPAE, Vol. 1, Doc. 111).

What in this article, (Lenard, 1900), could have sparked such a burst of enthusiasm? Here was Einstein's initiation to the photo-electric effect as it is now recognized. ${ }^{32}$ And it appears to be exactly the inverse process of the conversion of the energy of motion into light.

If, as is suggested, this is the birth of the idea of the light quantum, why did it take so long for Einstein to develop it and publish it? Part of the answer has already been given in that the sort of constructive argument given in (Einstein, 1905a) had to await the discovery of the inability of Maxwell's theory to account for energy fluctuations. Another part involves the bearing on Einstein's ongoing concerns with the electrodynamics of moving bodies. If radiation consists of light quanta, what role remains for the ether? If Einstein had already been impressed by the asymmetries of electromagnetic induction, the lightquantum hypothesis would fuel his investigation of the possibility that the velocity of light depends on the motion of the source. If he had not already been so impressed, it becomes pertinent to ask in explanations of Faraday induction, what grounds remain for saying the magnet, respectively, the current loop, is "in motion"? The correspondence with Mileva Marić establishes that something was abuzz later in $1901 .^{33}$

Finally, another part is that subsequent developments may have raised doubts in Einstein's mind about the viability of the light-quantum hypothesis. A pair of letters to Marić

\footnotetext{
${ }^{31}$ (Boltzmann, 1896), which Einstein had been studying at the time, gives recipes for evaluating integrals of the form appearing in these equations in connection with the presentation of Maxwell's velocity distribution.

${ }^{32}$ Prior to this, it had been unclear whether the electric current in the effect was the direct product of the incident radiation or due to some other factor, such as ionization of the gas inside the tube.

33 "I am working eagerly on an electrodynamics of moving bodies, which promises to become a capital paper. I wrote to you that I doubted the correctness of the ideas about relative motion. However, my qualms rested solely on a calculational error. Now I believe in them all the more" (Einstein to Mileva Marić, 17 December 1901, CPAE, Vol. 1, Doc. 128).

"Today ... I explained [to Kleiner] my ideas on the electrodynamics of moving bodies .... ... He advised me to publish my ideas on the electromagnetic theory of light for moving bodies together with the experimental method. He thought that the my suggested experimental method is the simplest conceivable and most appropriate" (Einstein to Mileva Marić, 19 December 1901, CPAE, Vol. 1, Doc. 130).
} 
earlier that April mention certain misgivings about Planck's theory of radiation, in particular, that Planck assumes "a completely definite sort of resonator (definite period and damping), an assumption I cannot quite accept" (Einstein to Mileva Marić, 10 April 1901, CPAE, Vol. 1, Doc. 97). Just why not, we will leave for the reader to speculate. The point that we would like to register is that, in the exchange of letters during this period, the order in which Einstein comments on articles from the Annalen der Physik, in all other cases, corresponds strictly to the chronological order in which they appeared. If his reading of Planck is not an exception to the rule, then what he had been reading in early April was not (Planck 1901a) and (Planck 1901b), but (Planck, 1900), in which Planck still considers Wien's spectral law to be strictly valid. Subsequently learning of Planck's law, which appears not to conform to the light-quantum hypothesis for low frequencies and high temperatures, Einstein, we suggest, may have reconsidered.

The crucial point is that, on this scenario, the light-quantum hypothesis in its initial incarnation is a thesis about the fundamental ontology of radiation. As Einstein pursued the hypothesis - or more properly, heuristic - in 1904 and 1905 - it was no longer necessarily a thesis about the underlying constitution of light, but, more modestly, one about the phenomenologically adequate description of light for various sorts of processes.

\section{Conclusion}

It is on this note that we wish to conclude. The year 1901 clearly belonged to the stage of Einstein's development which he later characterized as attempts "to discover the true laws by means of constructive efforts based on known fact" something which he gradually despaired of realizing (Einstein, 1975, p. 49). In the Autobiographical Notes this marks the transition to the discovery of the non-absolute character of simultaneity and the special theory of relativity, a theory which two years later he also characterized as a heuristic (Einstein, 1907, p. 206). It could have equally served to mark the turning point of the annus mirabilis. Perhaps the real miracle is salvaging success out of failure: discovering the limitations of the currently accepted foundations, and having nothing to replace them with, Einstein is nonetheless able to sift through the remains to secure, inductively, critical fixed points from which to carry on.

\section{References}

Boltzmann, L. (1877). Über die Beziehung zwischen dem zweiten Hauptsatze der mechanischen Wärmetheorie und der Wahrscheinlichkeitsrechnung resp. dem Sätzen über das Wärmegleichgewicht. Wiener Berichte 76, 373-435.

Boltzmann, L. (1896). Vorlesungen über Gastheorie, Part I. Leipzig: Johann Ambrosius Barth. 
CPAE (1987-2004). The collected papers of Albert Einstein. 9 Vols. Edited by J. Stachel et al. Princeton: Princeton University Press.

Einstein, A. (1903). Eine Theorie der Grundlagen der Thermodynamik. Annalen der Physik 11, 170-187. Also in (CPAE, Vol. 2, Doc. 4).

Einstein, A. (1904). Zur allgemeinen molekularen Theorie der Wärme. Annalen der Physik 14, 355-362. Also in (CPAE, Vol. 2, Doc. 5).

Einstein, A. (1905a). Über einen die Erzeugung und Verwandlung des Lichtes betreffenden heuristischen Gesichtspunkt. Annalen der Physik 17, 132-148. Also in (CPAE, Vol. 2, Doc. 14).

Einstein, A. (1905b). Eine neue Bestimmung der Moleküldimensionen. Bern: K. J. Wyss. Also in (CPAE, Vol. 2, Doc. 15). Slightly revised version published as (Einstein, 1906a).

Einstein, A. (1905c). Über die von der molekularkinetischen Theorie der Wärme geforderte Bewegung von in ruhenden Flüssigkeiten suspendierten Teilchen. Annalen der Physik 17, 549-560. Also in (CPAE, Vol. 2, Doc. 16).

Einstein, A. (1905d). Zur Elektrodynamik bewegter Körper. Annalen der Physik 17, 891-921. Also in (CPAE, Vol. 2, Doc. 23).

Einstein, A. (1906a). Eine neue Bestimmung der Moleküldimensionen. Annalen der Physik 19, 289-305.

Einstein, A. (1906b). Zur Theorie der Lichterzeugung und Lichtabsorption. Annalen der Physik 20, 199-206. Also in (CPAE, Vol. 2, Doc. 34).

Einstein, A. (1907). Bermerkungen zu der Notiz von Hrn. Paul Eherenfest: "Die Translation defomierbarer Elektronen und der Flachensatz." Annalen der Physik 23, 206-208. Also in (CPAE, Vol. 2, Doc. 44).

Einstein, A. (1954). Ideas and opinions. New York: Bonanza Books.

Einstein, A. (1979). Autobiographical notes: a centennial edition. Translated and edited by P. A. Schilpp. LaSalle: Open Court.

Einstein, A. (2005). Einstein's miraculous year, 2nd. J. Stachel (ed.). Princeton: Princeton University Press. 
Einstein, A. and Besso, M. (1972). Correspondance: 1903-1955. Edited and translated by P. Speziali. Paris: Hermann.

Einstein, A. and Marić, M. (1992). The love letters. Edited by J. Renn and R. Schulmann. Princeton: Princeton University Press.

Gearhart, C. A. (2002). Planck, the Quantum, and the Historians. Physics in Perspective 4: $170-215$.

Janssen, M. (1999). Rotation as the nemesis of Einstein's Entwurf theory. In H. Goenner et al. (eds.), The expanding worlds of general relativity (pp. 127-157). Boston: Birkhäuser.

Janssen, M. (2003). A glimpse behind the curtain of the wizard / Un coup d'œil derrière le rideau du magicien. In The Einstein-Besso manuscript: From special relativity to general relativity / Le manuscrit Einstein-Besso: De la relativité restreinte à la relativité générale. Paris: Scriptura and Aristophile.

Lenard, P. (1900). Erzeugung von Kathodenstrahlen durch ultraviolettes Licht. Annalen der Physik 2, 359-375.

Planck, M. (1900). Ueber irreversible Strahlungsvorgänge. Annalen der Physik 1, 69122.

Planck, M. (1901a). Ueber das Gesetz der Energieverteilung im Normalspectrum. Annalen der Physik 4, 553-563.

Planck, M. (1901b). Ueber die Elementarquanta der Materie und der Elektricität. Annalen der Physik 4, 564-566.

Renn, J. (2005). Einstein's invention of Brownian motion. Annalen der Physik 14, Supplement, 23-37.

Renn, J. (ed.) (forthcoming). The Genesis of General Relativity. 4 Vols. Dordrecht: Elsevier.

Rynasiewicz, R. (2000). The construction of the special theory: Some queries and considerations. In D. Howard and J. Stachel (eds.), Einstein: The formative years, 1879-1909 (pp. 159-201). Boston: Birkhuser.

Rynasiewicz, R. (2005). The optics and electrodynamics of 'On the electrodynamics of 
moving bodies'. Annalen der Physik 14, Supplement, 38-57.

Stachel, J. (2005). Introduction to the centenary edition. In (Einstein, 2005, pp. xvlxxii).

Seelig, C. (1956). Albert Einstein: A documentary biography. London: Staples Press.

Wien, W. (1983). Eine neue Beziehung der Strahlung schwarzer Körper zum zweiten Hauptsatz der Wärmetheorie. Königlich Preussisch Akadiemie der Wissenschaften zu Berlin: Sitzungsberichte, 9. Februar 1893, 55-62.

Wien, W. (1984). Temperatur und Entropie der Strahlung. Annalen der Physik und Chemie 52, 132-165.

Wien, W. (1986). Ueber die Energievertheilung im Emissionsspectrum eines schwarzen Körpers. Annalen der Physik und Chemie 58, 662-669. 